\title{
A New Hermite-Hadamard inequality for $h$-convex stochastic processes
}

\author{
Hüseyin Budak and Mehmet Zeki Sarikaya \\ Department of Mathematics, Faculty of Science and Arts, Düzce University, Düzce, Turkey \\ Received: 9 March 2019, Accepted: 27 May 2019 \\ Published online: 29 September 2019.
}

\begin{abstract}
Firstly, some new definitions which are the special cases of $h$-convex stochastic proceses are given. Then, we establish a new refinement of Hermite-Hadamard inequality for $h$-convex stochastic processes and give some special cases of this result.
\end{abstract}

Keywords: Hermite-Hadamard inequality, convex stochastic process, $h$-convex stochastic processes.

\section{Introduction}

The classical Hermite-Hadamard inequality which was first published in [5] gives us an estimate of the mean value of a convex function $f: I \rightarrow \mathbb{R}$,

$$
f\left(\frac{a+b}{2}\right) \leq \frac{1}{b-a} \int_{a}^{b} f(x) d x \leq \frac{f(a)+f(b)}{2}
$$

An account the history of this inequality can be found in [3]. Surveys on various generalizations and developments can be found in [2] and [9].

In 1980, Nikodem [10] introduced convex stochastic processes and investigated their regularity properties. In 1992, Skwronski [14] obtained some further results on convex functions.

Let $(\Omega, \mathscr{A}, P)$ be an arbitrary probability space. A function $X: \Omega \rightarrow \mathbb{R}$ is called a random variable if it is $\mathscr{A}$-measurable. A function $X: I \times \Omega \rightarrow \mathbb{R}$, where $I \subset \mathbb{R}$ is an interval, is called a stochastic process if for every $t \in I$ the function $X(t,$.$) is a random variable.$

Recall that the stochastic process $X: I \times \Omega \rightarrow \mathbb{R}$ is called $(i)$ continuous in probability in interval $I$, if for all $t_{0} \in I$ we have

$$
P-\lim _{t \rightarrow t_{0}} X(t, .)=X\left(t_{0}, .\right),
$$

where $P-\lim$ denotes the limit in probability. (ii) mean-square continuous in the interval $I$, if for all $t_{0} \in I$

$$
\lim _{t \rightarrow t_{0}} E\left[\left(X(t)-X\left(t_{0}\right)\right)^{2}\right]=0
$$

where $E[X(t)]$ denotes the expectation value of the random variable $X(t,$.$) .$

Obviously, mean-square continuity implies continuity in probability, but the converse implication is not true. 
Definition 1. Suppose we are given a sequence $\left\{\Delta^{m}\right\}$ of partitions, $\Delta^{m}=\left\{a_{m, 0}, \ldots, a_{m, n_{m}}\right\}$. We say that the sequence $\left\{\Delta^{m}\right\}$ is a normal sequence of partitions if the length of the greatest interval in the $n$-th partition tends to zero, i.e.,

$$
\lim _{m \rightarrow \infty} \sup _{1 \leq i \leq n_{m}}\left|a_{m, i}-a_{m, i-1}\right|=0
$$

Now we would like to recall the concept of the mean-square integral. For the definition and basic properties see [15].

Let $X: I \times \Omega \rightarrow \mathbb{R}$ be a stochastic process with $E\left[X(t)^{2}\right]<\infty$ for all $t \in I$. Let $[a, b] \subset I, a=t_{0}<t_{1}<t_{2}<\ldots<t_{n}=b$ be a partition of $[a, b]$ and $\Theta_{k} \in\left[t_{k-1}, t_{k}\right]$ for all $k=1, \ldots, n$. A random variable $Y: \Omega \rightarrow \mathbb{R}$ is called the mean-square integral of the process $X$ on $[a, b]$, if we have

$$
\lim _{n \rightarrow \infty} E\left[\left(\sum_{k=1}^{n} X\left(\Theta_{k}\right)\left(t_{k}-t_{k-1}\right)-Y\right)^{2}\right]=0
$$

for all normal sequence of partitions of the interval $[a, b]$ and for all $\Theta_{k} \in\left[t_{k-1}, t_{k}\right], k=1, \ldots, n$. Then, we write

$$
Y(\cdot)=\int_{a}^{b} X(s, \cdot) d s(\text { a.e. })
$$

For the existence of the mean-square integral it is enough to assume the mean-square continuity of the stochastic process $X$.

Throughout the paper we will frequently use the monotonicity of the mean-square integral. If $X(t, \cdot) \leq Y(t, \cdot)($ a.e. $)$ in some interval $[a, b]$, then

$$
\int_{a}^{b} X(t, \cdot) d t \leq \int_{a}^{b} Y(t, \cdot) d t \text { (a.e.). }
$$

Of course, this inequality is the immediate consequence of the definition of the mean-square integral.

Definition 2. We say that a stochastic processes $X: I \times \Omega \rightarrow \mathbb{R}$ is convex, if for all $\lambda \in[0,1]$ and $u, v \in I$ the inequality

$$
X(\lambda u+(1-\lambda) v, \cdot) \leq \lambda X(u, \cdot)+(1-\lambda) X(v, \cdot)(\text { a.e. })
$$

is satisfied. If the above inequality is assumed only for $\lambda=\frac{1}{2}$, then the process $X$ is Jensen-convex or $\frac{1}{2}-$ convex. A stochastic process $X$ is concave if $(-X)$ is convex. Some interesting properties of convex and Jensen-convex processes are presented in ([10], [15]).

Now, we present some results proved by Kotrys [6] about Hermite-Hadamard inequality for convex stochastic processes.

Lemma 1. If $X: I \times \Omega \rightarrow \mathbb{R}$ is a stochastic process of the form $X(t, \cdot)=A(\cdot) t+B(\cdot)$, where $A, B: \Omega \rightarrow \mathbb{R}$ are random variables, such that $E\left[A^{2}\right]<\infty, E\left[B^{2}\right]<\infty$ and $[a, b] \subset I$, then

$$
\int_{a}^{b} X(t, \cdot) d t=A(\cdot) \frac{b^{2}-a^{2}}{2}+B(\cdot)(b-a) \text { (a.e.). }
$$


Proposition 1. Let $X: I \times \Omega \rightarrow \mathbb{R}$ be a convex stochastic process and $t_{0} \in$ intI. Then there exist a random variable $A: \Omega \rightarrow \mathbb{R}$ such that $X$ is supported at $t_{0}$ by the process $A(\cdot)\left(t-t_{0}\right)+X\left(t_{0}, \cdot\right)$. That is

$$
X(t, \cdot) \geq A(\cdot)\left(t-t_{0}\right)+X\left(t_{0}, \cdot\right) \text { (a.e.) }
$$

for all $t \in I$.

Theorem 1. Let $X: I \times \Omega \rightarrow \mathbb{R}$ be Jensen-convex, mean-square continuous in the interval I stochastic process. Then for any $u, v \in I$ we have

$$
X\left(\frac{u+v}{2}, \cdot\right) \leq \frac{1}{v-u} \int_{u}^{v} X(t, \cdot) d t \leq \frac{X(u, \cdot)+X(v, \cdot)}{2} \text { (a.e.) }
$$

In [11], Sarıkaya et al. proved the following refinement of the inequality (3):

Theorem 2. If $X: I \times \Omega \rightarrow \mathbb{R}$ be Jensen-convex, mean-square continuous in the interval I stochastic process. Then for any $u, v \in I$ and for all $\lambda \in[0,1]$, we have

$$
X\left(\frac{u+v}{2}, \cdot\right) \leq h(\lambda) \leq \frac{1}{v-u} \int_{u}^{v} X(t, \cdot) d t \leq H(\lambda) \leq \frac{X(u, \cdot)+X(v, \cdot)}{2}
$$

where

$$
h(\lambda):=\lambda X\left(\frac{\lambda v+(2-\lambda) u}{2}, \cdot\right)+(1-\lambda) X\left(\frac{(1+\lambda) v+(1-\lambda) u}{2}, \cdot\right)
$$

and

$$
H(\lambda):=\frac{1}{2}(X(\lambda v+(1-\lambda) u, \cdot)+\lambda X(u, \cdot)+(1-\lambda) X(v, \cdot))
$$

In [1], Barraez et al. introduced the concept of $h$-convex stochastic process with following definition.

Definition 3. Let $h:(0,1) \rightarrow \mathbb{R}$ be a non-negative function, $h \neq 0$.we say that a stochastic process $X: I \times \Omega \rightarrow \mathbb{R}$ is an $h$-convex stochastic process if, for every $t_{1}, t_{2} \in I, \lambda \in(0,1)$, the following inequality is satisfied

$$
X(\lambda u+(1-\lambda) v, \cdot) \leq h(\lambda) X(u, \cdot)+h(1-\lambda) X(v, \cdot) \text { (a.e.) }
$$

Obviously, if we take $h(\lambda)=\lambda$ and $h(\lambda)=\lambda^{s}$ in (5), then the definition of $h$-convex stochastic process reduces to the definition of classical convex stochastic process [10] and $s$-convex stochastic process in the second sense [12] respectively. Moreover, A stochastic process $X: I \times \Omega \rightarrow \mathbb{R}$ is:

(1) Godunova-Levin stochastic process if, we take $h(\lambda)=\frac{1}{\lambda}$ in (5),

$$
X(\lambda u+(1-\lambda) v, \cdot) \leq \frac{X(u, \cdot)}{\lambda}+\frac{X(v, \cdot)}{1-\lambda}(\text { a.e. })
$$

(2) $P$-stochastic process if, we take $h(\lambda)=1$ in (5),

$$
X(\lambda u+(1-\lambda) v, \cdot) \leq X(u, \cdot)+X(v, \cdot) \text { (a.e.) }
$$

Authors proved the following Hermite-Hadamard inequality for $h$-convex stochastic process in [1].

Theorem 3. If $X: I \times \Omega \rightarrow \mathbb{R}$ Let be $h:(0,1) \rightarrow \mathbb{R}$ a non-negative function, $h \neq 0$ and $X: I \times \Omega \rightarrow \mathbb{R}$ a non negative, $h$-convex, mean square integrable stochastic process. For every $u, v \in I,(u<v)$, the following inequality is satisfied 
almost everywhere

$$
\frac{1}{2 h\left(\frac{1}{2}\right)} X\left(\frac{u+v}{2}, \cdot\right) \leq \frac{1}{v-u} \int_{u}^{v} X(t, \cdot) d t \leq[X(u, \cdot)+X(v, \cdot)] \int_{0}^{1} h(\lambda) d \lambda
$$

For more information and recent developments on Hermite-Hadamard type inequalities for stochastic process, please refer to ([1], [4], [6]-[8], [11]-[13], [16]). The aim of this paper is to establish an improvement of Hermite-Hadamard inequality for $h$-convex stochastic process.

\section{Main results}

Theorem 4. If $X: I \times \Omega \rightarrow \mathbb{R}$ Let be $h:(0,1) \rightarrow \mathbb{R}$ a non-negative function, $h \neq 0$ and $X: I \times \Omega \rightarrow \mathbb{R}$ a non negative, $h$-convex, mean square integrable stochastic process. For every $u, v \in I,(u<v)$, we have the following inequality

$$
\frac{1}{4\left[h\left(\frac{1}{2}\right)\right]^{2}} X\left(\frac{u+v}{2}, \cdot\right) \leq \Delta_{1} \leq \frac{1}{v-u} \int_{u}^{v} X(t, \cdot) d t \leq \Delta_{2} \leq[X(u, \cdot)+X(v, \cdot)]\left[\frac{1}{2}+h\left(\frac{1}{2}\right)\right] \int_{0}^{1} h(\lambda) d \lambda
$$

where

$$
\Delta_{1}:=\frac{1}{4 h\left(\frac{1}{2}\right)}\left[X\left(\frac{3 u+v}{4}, \cdot\right)+\left(\frac{u+3 v}{4}, \cdot\right)\right]
$$

and

$$
\Delta_{2}:=\left[\frac{X(u, \cdot)+X(v, \cdot)}{2}+X\left(\frac{u+v}{2}, \cdot\right)\right] \int_{0}^{1} h(\lambda) d \lambda
$$

Proof. Since $X: I \times \Omega \rightarrow \mathbb{R}$ is a $h$-convex stochastic process, we have

$$
\begin{aligned}
X\left(\frac{u+\frac{u+v}{2}}{2}, \cdot\right) & =X\left(\frac{\lambda u+(1-\lambda) \frac{u+v}{2}+(1-\lambda) u+\lambda \frac{u+v}{2}}{2}, \cdot\right) \\
& \leq h\left(\frac{1}{2}\right)\left[X\left(\lambda u+(1-\lambda) \frac{u+v}{2}, \cdot\right)+X\left((1-\lambda) u+\lambda \frac{u+v}{2}, \cdot\right)\right] .
\end{aligned}
$$

Integrating (9) from 0 to 1 with respect to $\lambda$, we get

$$
\begin{aligned}
X\left(\frac{3 u+v}{4}, \cdot\right) & \leq h\left(\frac{1}{2}\right)\left[\int_{0}^{1} X\left(\lambda u+(1-\lambda) \frac{u+v}{2}, \cdot\right) d \lambda+\int_{0}^{1} X\left((1-\lambda) u+\lambda \frac{u+v}{2}, \cdot\right) d \lambda\right] \\
& \leq h\left(\frac{1}{2}\right)\left[\frac{2}{v-u} \int_{u}^{\frac{u+v}{2}} X(t, \cdot) d t+\frac{2}{v-u} \int_{u}^{\frac{u+v}{2}} X(t, \cdot) d t\right] \\
& =\frac{4 h\left(\frac{1}{2}\right)}{v-u} \int_{u}^{\frac{u+v}{2}} X(t, \cdot) d t .
\end{aligned}
$$

That is,

$$
\frac{1}{4 h\left(\frac{1}{2}\right)} X\left(\frac{3 u+v}{4}, \cdot\right) \leq \frac{1}{v-u} \int_{u}^{\frac{u+v}{2}} X(t, \cdot) d t
$$


Since $X$ is a $h$-convex stochastic process, we also have

$$
\begin{aligned}
X\left(\frac{\frac{u+v}{2}+v}{2}, \cdot\right) & =X\left(\frac{\lambda \frac{u+v}{2}+(1-\lambda) v+(1-\lambda) \frac{u+v}{2}+\lambda v}{2}, \cdot\right) \\
& \leq h\left(\frac{1}{2}\right)\left[X\left(\lambda \frac{u+v}{2}+(1-\lambda) v, \cdot\right)+X\left((1-\lambda) \frac{u+v}{2}+\lambda v, \cdot\right)\right]
\end{aligned}
$$

Integrating (12) from 0 to 1 with respect to $\lambda$, we get

$$
\begin{aligned}
X\left(\frac{u+3 v}{4}, \cdot\right) & \leq h\left(\frac{1}{2}\right)\left[\int_{0}^{1} X\left(\lambda \frac{u+v}{2}+(1-\lambda) v, \cdot\right) d \lambda+\int_{0}^{1} X\left((1-\lambda) \frac{u+v}{2}+\lambda v, \cdot\right) d \lambda\right] \\
& \leq h\left(\frac{1}{2}\right)\left[\frac{2}{v-u} \int_{\frac{u+v}{2}}^{v} X(t, \cdot) d t+\frac{2}{v-u} \int_{\frac{u+v}{2}}^{v} X(t, \cdot) d t\right] \\
& =\frac{4 h\left(\frac{1}{2}\right)}{v-u} \int_{\frac{u+v}{2}}^{v} X(t, \cdot) d t
\end{aligned}
$$

i.e.

$$
\frac{1}{4 h\left(\frac{1}{2}\right)} X\left(\frac{u+3 v}{4}, \cdot\right) \leq \frac{1}{v-u} \int_{\frac{u+v}{2}}^{v} X(t, \cdot) d t .
$$

Summing inequalities (11) and (13), we obtain

$$
\Delta_{1}=\frac{1}{4 h\left(\frac{1}{2}\right)}\left[X\left(\frac{3 u+v}{4}, \cdot\right)+\left(\frac{u+3 v}{4}, \cdot\right)\right] \leq \frac{1}{v-u} \int_{u}^{v} X(t, \cdot) d t
$$

which finishes the proof of second inequality in (8).

Applying the Hermite-Hadamard inequality for $h$-convex stochastic process (Theorem 3), we have

$$
\begin{aligned}
\frac{1}{v-u} \int_{u}^{v} X(t, \cdot) d t & =\frac{1}{2}\left[\frac{2}{v-u} \int_{u}^{\frac{u+v}{2}} X(t, \cdot) d t+\frac{2}{v-u} \int_{\frac{u+v}{2}}^{v} X(t, \cdot) d t\right] \\
& \leq \frac{1}{2}\left[\left[X(u, \cdot)+X\left(\frac{u+v}{2}, \cdot\right)\right] \int_{0}^{1} h(\lambda) d \lambda\right]+\frac{1}{2}\left[\left[X\left(\frac{u+v}{2}, \cdot\right)+X(v, \cdot)\right] \int_{0}^{1} h(\lambda) d \lambda\right] \\
& =\left[\frac{X(u, \cdot)+X(v, \cdot)}{2}+X\left(\frac{u+v}{2}, \cdot\right)\right] \int_{0}^{1} h(\lambda) d \lambda \\
& =\Delta_{2} .
\end{aligned}
$$

This completes the proof of third inequality in (8). 
For the first inequality, using the $h$-convexity of $X$, we have

$$
\begin{aligned}
\frac{1}{4\left[h\left(\frac{1}{2}\right)\right]^{2}} X\left(\frac{u+v}{2}, \cdot\right) & =\frac{1}{4\left[h\left(\frac{1}{2}\right)\right]^{2}} X\left(\frac{1}{2} \frac{3 u+v}{2}+\frac{1}{2} \frac{u+3 v}{4}, \cdot\right) \\
& \leq \frac{1}{4\left[h\left(\frac{1}{2}\right)\right]^{2}}\left[h\left(\frac{1}{2}\right) X\left(\frac{3 u+v}{2}, \cdot\right)+h\left(\frac{1}{2}\right) X\left(\frac{u+3 v}{4}, \cdot\right)\right] \\
& =\Delta_{1} .
\end{aligned}
$$

Finally,

$$
\begin{aligned}
\Delta_{2} & =\left[\frac{X(u, \cdot)+X(v, \cdot)}{2}+X\left(\frac{u+v}{2}, \cdot\right)\right] \int_{0}^{1} h(\lambda) d \lambda \\
& \leq\left[\frac{X(u, \cdot)+X(v, \cdot)}{2}+h\left(\frac{1}{2}\right)[X(u, \cdot)+X(v, \cdot)]\right] \int_{0}^{1} h(\lambda) d \lambda \\
& =[X(u, \cdot)+X(v, \cdot)]\left[\frac{1}{2}+h\left(\frac{1}{2}\right)\right] \int_{0}^{1} h(\lambda) d \lambda .
\end{aligned}
$$

This completes completely the proof of the Theorem.

Remark. Under assumption of Theorem 4 with $h(t)=t$, we have

$$
X\left(\frac{u+v}{2}, \cdot\right) \leq \Delta_{1} \leq \frac{1}{v-u} \int_{u}^{v} X(t, \cdot) d t \leq \Delta_{2} \leq \frac{X(u, \cdot)+X(v, \cdot)}{2}
$$

where

$$
\Delta_{1}:=\frac{1}{2}\left[X\left(\frac{3 u+v}{4}, \cdot\right)+\left(\frac{u+3 v}{4}, \cdot\right)\right]
$$

and

$$
\Delta_{2}:=\frac{1}{2}\left[\frac{X(u, \cdot)+X(v, \cdot)}{2}+X\left(\frac{u+v}{2}, \cdot\right)\right] .
$$

This inequality is a special case of the Theorem 2 with $\lambda=\frac{1}{2}$.

Corollary 1. Under assumption of Theorem 4 with $h(t)=t^{s}$, we have the refinement Hermite-Hadamard inequality for s-convex stochastic processes in the second sense

$$
2^{2 s-2} X\left(\frac{u+v}{2}, \cdot\right) \leq \Delta_{1} \leq \frac{1}{v-u} \int_{u}^{v} X(t, \cdot) d t \leq \Delta_{2} \leq[X(u, \cdot)+X(v, \cdot)]\left[\frac{1}{2}+\frac{1}{2^{s}}\right] \frac{1}{s+1}
$$

where

$$
\Delta_{1}=2^{s-2}\left[X\left(\frac{3 u+v}{4}, \cdot\right)+\left(\frac{u+3 v}{4}, \cdot\right)\right]
$$

and

$$
\Delta_{2}=\left[\frac{X(u, \cdot)+X(v, \cdot)}{2}+X\left(\frac{u+v}{2}, \cdot\right)\right] \frac{1}{s+1}
$$


Corollary 2. Under assumption of Theorem 4 with $h(t)=1$, we have the following Hermite-Hadamard type inequality for $P$-stochastic processes

$$
\frac{1}{4} X\left(\frac{u+v}{2}, \cdot\right) \leq \Delta_{1} \leq \frac{1}{v-u} \int_{u}^{v} X(t, \cdot) d t \leq \Delta_{2} \leq \frac{3}{2}[X(u, \cdot)+X(v, \cdot)]
$$

where

$$
\Delta_{1}=\frac{1}{4}\left[X\left(\frac{3 u+v}{4}, \cdot\right)+\left(\frac{u+3 v}{4}, \cdot\right)\right]
$$

and

$$
\Delta_{2}=\left[\frac{X(u, \cdot)+X(v, \cdot)}{2}+X\left(\frac{u+v}{2}, \cdot\right)\right] .
$$

Corollary 3. Under assumption of Theorem 4 with $h(t)=\frac{1}{t}$, we have the following Hermite-Hadamard type inequality for Godunova-Levin stochastic processes

$$
\frac{1}{16} X\left(\frac{u+v}{2}, \cdot\right) \leq \Delta \leq \frac{1}{v-u} \int_{u}^{v} X(t, \cdot) d t
$$

where

$$
\Delta=\frac{1}{8}\left[X\left(\frac{3 u+v}{4}, \cdot\right)+\left(\frac{u+3 v}{4}, \cdot\right)\right] .
$$

\section{Competing interests}

The authors declare that they have no competing interests.

\section{Authors' contributions}

All authors have contributed to all parts of the article. All authors read and approved the final manuscript.

\section{References}

[1] D. Barraez, L. Gonzalez, N. Merentes and A. M. Moros, On h-convex stochastic process, Mathematica Aeterna,5(4), 2015, 571581.

[2] S. S. Dragomir and R.P. Agarwal, Two inequalities for differentiable mappings and applications to special means of real numbers and to trapezoidal formula, Appl. Math. lett., 11(5) (1998), 91-95.

[3] S. S. Dragomir and C. E. M. Pearce, Selected Topics on Hermite-Hadamard Inequalities and Applications, RGMIA Monographs, Victoria University, 2000.

[4] L. Gonzalez, N. Merentes, and M. Valera-Lopez, Some estimates on the Hermite-Hadamard inequality through convex and quasiconvex stochastic processes, Mathematica Aeterna, 5(5), 2015, 745-767.

[5] J. Hadamard, Etude sur les proprietes des fonctions entieres et en particulier d'une fonction consideree par Riemann, J. Math. Pures Appl., 58(1893), 171-215.

[6] D. Kotrys, Hermite-Hadamard inequality for convex stochastic processes, Aequat. Math. 83 (2012), 143-151.

[7] S. Maden, M. Tomar and E. Set, Hermite-Hadamard type inequalities for s-convex stochastic processes in first sense, Pure and Applied Mathematics Letters, Volume 2015, 1-7.

[8] J. Materano, N. Merentes and M. Valera-Lopez, Some estimates on the Simpson's type inequalities through s-convex and quasiconvex stochastic processes, Mathematica Aeterna, 5(5), 2015, 673-705. 
[9] D. S. Mitrinovic, J. E. Pečarič, and A. M. Fink, Classical and new inequalities in analysis, ser. Math. Appl. (East European Ser.). Dordrecht: Kluwer Academic Publishers Group, 1993, vol. 61

[10] K. Nikodem, On convex stochastic processes, Aequat. Math. 20, 184-197 (1980).

[11] M. Z. Sarikaya, H. Yaldiz and H. Budak, Some integral inequalities for convex stochastic process, Acta Mathematica Universitatis Comenianae, Vol. LXXXV, 1 (2016), 155-164.

[12] E. Set, M. Tomar and S. Maden, Hermite-Hadamard type inequalities for s-convex stochastic processes in the second sense, Turkish Journal of Analysis and Number Theory, 2(6), 2014: 202-207. doi: 10.12691/tjant-2-6-3.

[13] E. Set, M. Z. Sarikaya and M. Tomar, Hermite-Hadamard type inequalities forcoordinates convex stochastic processes, Mathematica Aeterna, 5(2), 2015, 363 - 382.

[14] A. Skowronski, On some properties of J-convex stochastic processes, Aequat. Math. 44, 249-258 (1992).

[15] K. Sobczyk, Stochastic differential equations with applications to physics and engineering, Kluwer, Dordrecht (1991).

[16] M. Tomar, E. Set and S. Maden, Hermite-Hadamard type inequalities for log - convex stochastic processes, Journal of New Theory, $2,2015,23-32$. 